\title{
Pacientes com carcinoma de células escamosas - relação do tratamento com o prognóstico
}

\author{
Relationship between Treatment and Prognosis in Patients with Squamous Cell Carcinoma \\ Mariana Teixeira Tillmann', Anelize de Oliveira Campello Felix', Cristina Gevehr Fernandes', \\ Sabrina de Oliveira Capella' ${ }^{1}$ Eduardo Negri Mueller² \& Márcia de Oliveira Nobre' ${ }^{1}$
}

\begin{abstract}
Background: Squamous cell carcinoma (SCC) is a malignant cutaneous neoplasm which occurs frequently in small animals. Histopathology or fine needle aspiration cytology is necessary to confirm diagnosis; macroscopic diagnosis is not possible since the lesions are very similar to others of distinct etiologies. Owing to the fact that it is a neoplasm, diagnosis and treatment are usually not well accepted by owners, especially since it can cause esthetic changes to the animal and adjunct treatments can cause unwanted side effects. The objective of this study was to report clinical cases of SCC with distinct tumor subtypes and relate the recommended treatment with prognosis of patients.

Case: Two dogs and one cat with SCC that were subjected to physical and dermatological examination, and tested negative for sporotrichosis, cryptococcosis, and manges. Dog 1 (male, 9-year-old, yellow Labrador retriever) exhibited inspiratory dyspnea for three weeks owing to an ulcerative hemorrhagic lesion on the nose. In view of the site of the lesion, a radiographic exam was requested, and the bony portion of the septum was found to be compromised. Cat 2 (female, 10-year-old, bicolor, mixed-breed cat) exhibited a focal, punctate, ulcerated, hemorrhagic lesion on the nose. Dog 3 (female, 10-year-old, white Dogo Argentino) exhibited several ulcerative lesions, and papulae, plaques and comedones on the ventral region of the abdomen.Treatment for deep pyoderma and comedone syndrome were initially instituted; on follow-up, more ulcerative lesions were present, which prompted the inclusion of neoplasm as a differential diagnosis. In cases 1 and 3, histopathology was performed with diagnosis of undifferentiated and differentiated SCC, respectively. In both cases, surgical margins were compromised after resection of the lesion. In case 2, high malignancy SCC was diagnosed with cytology. Use of sunscreen was recommended in all cases, as well as avoidance of sun exposure during the hours not recommended by the National Cancer Institute. In case 1, a nosectomy with partial resection of the nasal septum was recommended as well as chemotherapy with intra-tumoral administration of carboplatin $(1.5 \mathrm{mg} / \mathrm{cm} 3$ of lesion) and vincristine sulfate $(0.05 \mathrm{mg} /$ $\mathrm{kg} / \mathrm{IV} /$ week) every 15 days.However, the owner opted for the nosectomy alone with one month of chemotherapy with vincristine sulfate. In case 2 , polychemotherapy was instituted using daunorubicin $(4 \mathrm{mg} / \mathrm{kg} / \mathrm{IV} /$ week $)$ and vincristine sulfate $(0.05 \mathrm{mg} / \mathrm{kg} / \mathrm{IV} /$ week $)$ every 15 days for four weeks, with maintenance for six weeks, with total remission of the lesion at the end of treatment. In case 3, eight intralesional chemotherapy sessions were performed (carboplatin $-1.5 \mathrm{mg} / \mathrm{cm} 3 \mathrm{of}$ lesion) every 15 days; maintenance was kept for two months with biweekly administration. The patient exhibited actinic lesions on the third month after the protocol was suspended, which was treated with isotretinoin $(3 \mathrm{mg} / \mathrm{kg} / \mathrm{day} \mathrm{PO})$ until remission; reduction of solar exposure was recommended. The animal in case 1 exhibited intense dyspnea three months after the end of treatment and the lesion was incompatible with preservation of quality of life and euthanasia was performed. In the other two cases, patients were followed-up annually and remained in remission throughout life.

Discussion: Adherence to proposed treatment by the owner is directly related to a better prognosis. On the punctate and focal lesion in the cat, administrations of daunorubicin ( $4 \mathrm{mg} / \mathrm{kg} / \mathrm{IV} /$ week) and vincristine sulfate $(0.05 \mathrm{mg} / \mathrm{kg} / \mathrm{IV} / \mathrm{week})$ in a total of 10 cycles resulted in an excellent prognosis. On the dog with multifocal lesions, partial resection and intralesional administration of carboplatin $(1.5 \mathrm{mg} / \mathrm{lesion} / \mathrm{cm} 3)$ in a total of eight biweekly applications following maintenance for two months resulted in a good prognosis.
\end{abstract}

Keywords: cat, chemotherapy, dog, oncology. 


\section{INTRODUÇÃO}

O carcinoma de células escamosas (CCE) é um neoplasma cutâneo maligno originário dos queratinócitos, comum em pequenos animais. O comportamento biológico do CCE é invasivo, com crescimento lento e baixo potencial para metástases. As lesões macroscópicas costumam ser proliferativas, hiperêmicas, crostosas, podendo evoluir para úlceras [6]. Devido a essas características o diagnóstico diferencial de enfermidades fúngicas e bacterianas, afecções da cavidade oral, lesões traumáticas e outros neoplasmas deve ser realizado, por citologia ou histopatologia (padrão-ouro) para obtenção do diagnóstico definitivo[3,5,6,9].

Considerando que esse neoplasma é um desafio para o veterinário desde o diagnóstico até o tratamento, pois para a realização do diagnóstico definitivo é necessária a interação de profissionais de diversas áreas. Além do fato que o tutor tem naturalmente dificuldade em aceitar o diagnóstico devido ao prognóstico favorável estar vinculado a sessões de quimioterapia elou afins que podem causar possíveis efeitos colaterais e com cirurgias onde podem ocorrem mudanças estéticas do seu animal $[5,9]$. O presente estudo objetiva relatar casos clínicos de CCE com subtipos tumorais distintos e relacionar o tratamento preconizado com o prognóstico dos pacientes.

\section{CASO}

Foram estudados três casos de carcinomas de células escamosas, sendo que dois foram em cães e um felino. Todos os animais foram submetidos a exame clínico geral, seguido de exame específico dermatológico. Sendo realizado raspado cutâneo em casos de lesões crostosas para avaliação de presença de ectoparasitas (Demodex canis, Sarcoptes scabei, Notoedres cati e Cheyletiella spp.) e imprint ou citoaspirado por agulha fina (CAAF) em casos de lesões ulceradas. Também foi realizada cultura e antibiograma quando havia suspeita de infecção bacteriana, para todos os casos foi realizado diagnóstico diferencial para esporotricose e criptococose, sendo os exames micológicos negativo para todos os casos. Os tutores foram questionados sobre lesões dermatológicas em humanos contactantes, os quais negaram nos três casos avaliados. No caso 1 onde a lesão era na face, podendo ter havido invasão óssea foi requisitado exame radiográfico da face.

Nos cães que possuíam lesões de mais de $2 \mathrm{~cm}$ de diâmetro foi realizada biópsia e encaminhado o ma- terial para a análise histopatológica enquanto no felino, cuja lesão era de aproximadamente $1 \mathrm{~cm}$ de diâmetro, foi utilizada a punção aspirativa por agulha fina. Os exames histopatológico e/ou citológico confirmaram o diagnóstico de carcinoma de células escamosas (CCE) em todos os casos. Os referidos casos estão detalhados abaixo, com os respectivos tratamentos instituídos. Nos cães onde foi realizado exérese tumoral, havendo presença de margem cirúrgica comprometida após o procedimento, a quimioterapia foi instituída após a cicatrização cirúrgica. Previamente as sessões de quimioterapia foi realizado hemograma e exame clínico, em todos os pacientes, para avaliar o estado clínico geral, não havendo alterações nos exames consecutivos. Como todos os animais tinham acesso à rua foi indicada a aplicação de fotoprotetores, e que estes evitassem a luz solar nos horários não recomendados pelo Instituto Nacional do Câncer.

Caso 1: canino, da raça labrador de pelagem baia, macho com nove anos de idade, vivia em ambiente com terra e exposição solar continua e havia contato com pombos. $\mathrm{O}$ animal apresentava uma lesão ulcerativa e hemorrágica na região da trufa há três semanas e consequente dispneia inspiratória. Na radiografia da face foi constatado possível comprometimento ósseo do septo nasal. Na análise histopatológica observou-se proliferação de queratinócitos atípicos, organizados em grandes trabéculas ou ninhos, que continham queratinização irregular ou necrose em seu interior, sendo confirmado como CCE indiferenciado (Figura 1B). Considerando o diagnóstico foi recomendado a realização de nosectomia com ressecção parcial do septo nasal e quimioterapia com carboplatina intra-tumoral (Tecnocarb ${ }^{\circledR 1}-1,5 \mathrm{mg} / \mathrm{cm}^{3}$ de lesão) e sulfato de vincristina (Tecnocris ${ }^{\circledR 1}-0,05 \mathrm{mg} / \mathrm{kg} / \mathrm{IV} / \mathrm{semana}$ ) a cada 15 dias com acompanhamento do caso para determinar o ciclo quimioterápico. No entanto o tutor optou pela

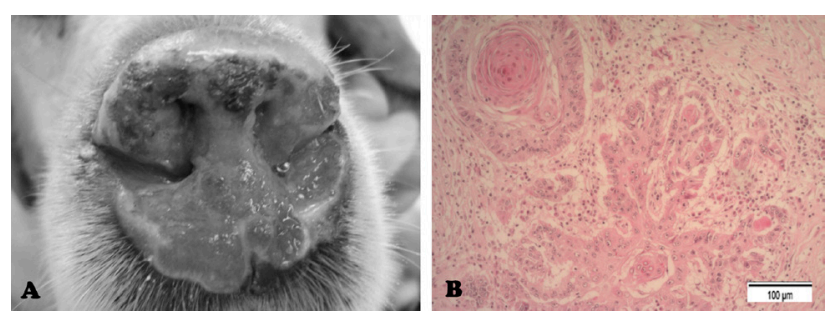

Figura 1. A- Lesão pós a nosectomia com posterior cauterização em um cão, labrador, baio, macho com nove anos de idade, que previamente ao procedimento cirúrgico apresentava lesão ulcerativa e hemorrágica na região da trufa há três semanas. B- Lesão histológica de carcinoma de células escamosas indiferenciado onde observa-se proliferação de queratinócitos atípicos, organizados em grandes trabéculas ou ninhos, que continham queratinização irregular ou necrose em seu interior [Barra $=100 \mu \mathrm{m}$ ]. 
realização apenas da nosectomia com posterior cauterização (Figura 1A) e pela quimioterapia com sulfato de vincristina (Tecnocris $\left.{ }^{\circledR}\right)^{1}$ a cada 15 dias por um mês. A argumentação referida foi a indisponibilidade financeira e o não concordar com a alteração estética do seu animal. Visto que o laudo histopatológico da amostra determinou que haviam margens comprometidas, foi recomendado novamente ao tutor que a utilização da carboplastina intra-tumoral $\left(\text { Tecnocarb }^{\circledR}\right)^{1}$ promoveria um prognóstico favorável. No entanto o mesmo manteve a opinião, mas ficou satisfeito com a melhora da qualidade de vida do seu cão e realizou um mês de quimioterapia sendo que durante esse período o paciente não teve recidiva macroscopicamente. Após três meses do fim do tratamento o canino apresentou recidiva, apresentando dispneia intensa como a lesão era incompatível com a qualidade de vida do paciente, foi realizada a eutanásia.

Caso 2: felino, fêmea, sem raça definida (SRD), bicolor, de 10 anos de idade, apresentava uma lesão puntiforme ulcerada e hemorrágica no focinho (Figura 2A), o paciente era domiciliado porém tinha contato com a rua. Foi realizado imprint da lesão a qual sugeriu neoplasma. Devido a isso foi realizado citoaspirado por agulha fina, demonstrando presença de agregados de células epiteliais escamosas, apresentando citoplasma bem delimitado, moderadamente basofílico, com presença de vacúolos perinucleares, anisocitose, anisocariose discreta, cromatina grosseira, nucléolos inconspícuos, presença de multinucleação e com figuras de empeiripolese, sendo assim confirmado carcinoma de células escamosas de alta malignidade (Figura 2B). Nesse paciente foi instituído o protocolo quimioterápico com daunorrubicina (Daunoblastina ${ }^{\circledR 2}$ - $4 \mathrm{mg} / \mathrm{kg} / \mathrm{IV} / \mathrm{semana}$ ) e sulfato de vincristina (Tec-
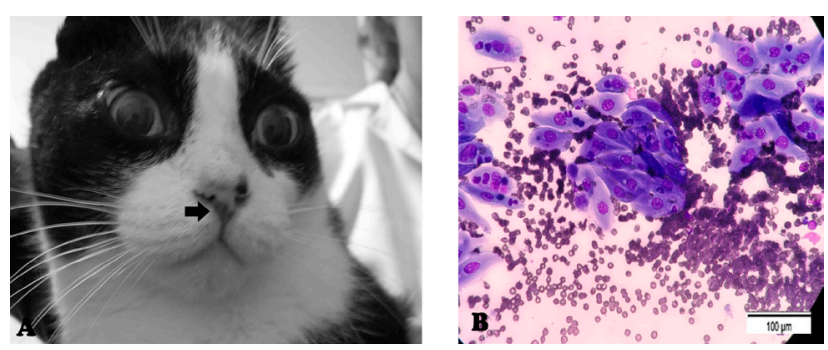

Figura 2. A- Lesão puntiforme, ulcerada e hemorrágica no focinho de um felino, fêmea, sem raça definida, bicolor, de 10 anos de idade. B- Lesão citológica de carcinoma de células escamosas de alta malignidade demonstrando presença de agregados de células epiteliais escamosas, apresentando citoplasma bem delimitado, moderadamente basofílico, com presença de vacúolos perinucleares, anisocitose, anisocariose discreta, cromatina grosseira, nucléolos inconspícuos, presença de multinucleação e com figuras de empeiripolese [Barra $=100 \mu \mathrm{m}]$. (Fonte Figura 2B - Thaís Rapachi Mann). nocris ${ }^{\circledR 1}-0,05 \mathrm{mg} / \mathrm{kg} / \mathrm{IV} / \mathrm{semana}$ ), a cada 15 dias por quatro semanas e a manutenção por seis semanas e foi acompanhado anualmente se mantendo em remissão do neoplasma durante toda a vida.

Caso 3: cão, fêmea, da raça dogo argentino, pelagem branca, de 10 anos, que apresentava grande quantidade de lesões ulcerativas, com presença de pápulas, placas e comedões na região ventral do abdômen. O raspado cutâneo e imprint foram negativos para ectoparasitas e fungos, no bacteriológico houve presença de Pseudomonas aeroginosa. Dessa forma havendo a suspeita de piodermatite profunda e síndrome dos comedões, sendo instituído o tratamento de acordo com o antibiograma (ciprofloxacina-Cipro ${ }^{\circledR 3}$ - $15 \mathrm{mg} / \mathrm{kg}$ a cada $12 \mathrm{~h}$ por 21 dias). Após 15 dias houve reavaliação do paciente e foi observado que houve melhora na síndrome dos comedões, porém a presença de pápulas persistia e algumas lesões haviam evoluído para úlceras. Considerando a evolução do quadro clínico foi realizado imprint nas lesões ulceradas. No imprint haviam bactérias, células inflamatórias, fibrina e células cornificadas, e no citoaspirado grupos celulares grandes com núcleos de cromatina grosseira, o citoplasma de algumas células apresentava-se levemente caudado, sugerindo CCE. Frente a suspeita optou-se pela ressecção cirúrgica da maior quantidade possíveis de lesões e encaminhamento para avaliação histopatológica, sendo que na histologia foi observado proliferação de queratinócitos neoplásicos, organizados em trabéculas finas, muito invasivos e com formação de pérolas de queratina sendo dessa foram diagnosticado um CCE diferenciado com margens cirúrgicas comprometidas (Figura 3B).Foi recomendado a utilização de carboplatina $\left(\text { Tecnocarb }^{\circledR}\right)^{1}$ na dose de 1,5 $\mathrm{mg} / \mathrm{cm}^{3}$ de lesão a cada 15 dias até a remissão das massas neoplásicas. Ao todo foram realizadas oito sessões com a aplicação do quimioterápico, pois somente nesse período houve a remissão total das lesões. Após esse período o tratamento prosseguiu como manutenção, com aplicação quinzenais durante 2 meses. Nos dois meses seguintes a suspensão do protocolo quimioterápico o animal não apresentava lesões recidivantes. No entanto após dois meses da suspensão do protocolo quimioterápico, o paciente apresentou esporadicamente presença de lesões actínicas. Devido, a presença dessas lesões foi recomendado que, além do uso do protetor solar, o paciente fosse tratado com isotretinoína (Roacutan ${ }^{\circledR 4} 3 \mathrm{mg} / \mathrm{kg} / \mathrm{dia}-\mathrm{VO}$ ) durante 4 ou 6 semanas, até a remissão das lesões. Além de mantido em um pátio totalmente coberto com malha 
de sombreamento de $80 \%\left(\text { Polysack }^{\circledR}\right)^{5}$ impedindo dessa forma a exposição intensa a luz solar. O paciente foi acompanhado anualmente não havendo recidiva do neoplasma durante a sua vida, apenas a presença das lesões actínicas esporadicamente que foram controladas com o tratamento e manejo instituído.

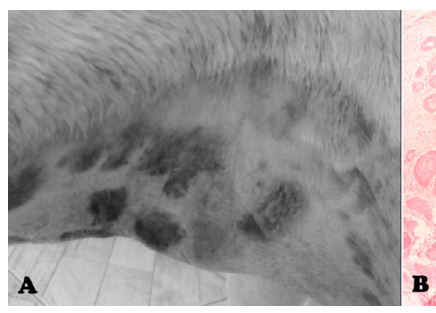

Figura 3. A- Pós operatório da exérese de parte das lesões ulcerativas, com presença de pápulas, placas e comedões na região ventral do abdômen de um cão, fêmea, da raça dogo argentino, pelagem branca, de 10 anos. B- Lesão histológica de carcinoma de células escamosas diferenciado sendo observado proliferação de queratinócitos neoplásicos, organizados em trabéculas finas, muito invasivos e com formação de pérolas de queratina [Barra de $50 \mu \mathrm{m}$ ].

\section{DISCUSSÃO}

Com relação aos protocolos quimioterápicos a aplicação somente da daunorrubicina e vincristina foram eficientes em lesão única e puntiforme, assim como a carboplatina intratumoral com exérese parcial dos neoplasmas no caso de lesões múltiplas. O subtipo tumoral nem sempre determina o estadiamento, principalmente quando vinculado ao diagnóstico precoce, pois quando o sítio anatômico da lesão possibilita exérese e o tutor adere a cirurgia recomendada o prognóstico se torna favorável, devido a possibilidade de margens cirúrgicas livres, e consequente garantia de qualidade de vida [9].

No caso 1, o protocolo recomendado incluía um antineoplásico de ação local, que seria mais eficiente em manter o local livre das células neoplásicas [7,9], vinculado a vincristina que estacionaria o crescimento neoplásico mas não iria ocasionar a morte das células cancerígenas [2]. No caso 2, considerando que o subtipo tumoral e pela lesão ser focal e pequena optou-se por realizar apenas uma poliquimioterapia, sem exérese cirúrgica. O protocolo com daunorrubicina e vincristina teve como intenção associar o efeito da vincristina com um quimioterápico do grupo das antraciclinas que oca- siona danos ao DNA da célula ocasionando sua morte, similar a ação da doxorrubicina [1,2]. No caso 3, onde as margens cirúrgicas estavam comprometidas foi realizada a administração de quimioterápico intralesional, pois permite uma alta concentração da droga no sítio da lesão, aumentando a sua atividade anti-tumoral e diminuindo a toxicidade [7,9]. Considerando que as lesões actínicas não são consideradas recidivantes por serem pré-neoplásicas podendo evoluir para um carcinoma epidermoíde podendo indicar uma predisposição genética a enfermidade [8]. Demonstrando assim a eficiência do protocolo quimioterápico, quando não foi possível a exérese total do neoplasma, ocasionando a remissão tumoral com ausência de efeitos colaterais [9]. No entanto isso foi possível com a ação conjunta de impedir a exposição intensa a luz solar, que é o principal fator desencadeante do CCE [6].

Esses relatos de casos nos demonstram que o câncer tem cura e existem possibilidades de tratamento e com manutenção da qualidade de vida, mesmo quando o animal tem predisposição genética a enfermidade [8]. Além de ser possível manter os pets no seu ambiente rotineiro, com apenas adaptações para diminuir a exposição solar. E que esta enfermidade é um desafio clínico psicológico no qual os veterinários enfrentam diariamente, tornando-se importante uma troca de experiência no meio veterinário, assim como acontece entre os médicos oncologistas [4,5].

\section{MANUFACTURERS}

${ }^{1}$ Zodiac Produtos Farmacêuticos S/A. Monções, SP, Brazil.

${ }^{2}$ Laboratórios Pfizer Ltda. Guarulhos, SP, Brazil.

${ }^{3}$ Bayer S.A. Belford Roxo, RJ, Brazil.

${ }^{4}$ Roche Químicos e Farmacêuticos S.A. Rio de Janeiro, RJ, Brazil. ${ }^{5}$ Polysack Copyright. São Paulo, SP, Brazil.

Funding. A CAPes e ao CNPq pela concessão de bolsa e auxílio financeiro.

Acknowledgements. Ao Centro de Saúde Animal Amigos para Sempre pela cooperação.

Ethical approval. Este projeto foi submetido e aprovado no comitê de ética da Universidade Federal de Pelotas sob o processo 23110.006104/2013-17.

Declaration of interest. The authors report no conflicts of interest. The authors alone are responsible for the content and writing of the paper.

\section{REFERENCES}

1 Almeida V.L., Leitão A., Reina L.C.B., Montanari C.A., Donnici C.L. \& Lopes M.T.P. 2005. Câncer e agentes antineoplásicos ciclo-celular específicos e ciclo-celular não específicos que interagem com o DNA: uma introdução. Química Nova. 28(1): 118-129. 
M.T.Tillmann, A.O.C. Felix, C.G. Fernandes, S.O. Capella, E.N. Mueller \& M.O. Nobre. 2017. Pacientes com carcinoma de células escamosas - relação do tratamento com o prognóstico. Acta Scientiae Veterinariae. 45(Suppl 1): 220.

2 Andrade S.F. 2008. Terapêutica Antineoplásica. In: Andrade S.F. (Ed). Manual de Terapêutica Veterinária. 3.ed. São Paulo: Roca, pp.201-224.

3 Bonfanti U., Bertazzolo W., Gracis M., Roccabianca P., Romanelli G., Palermo G. \& Zini E. 2014. Diagnostic value of cytological analysis of tumours and tumour-like lesions of the oral cavity in dogs and cats: A prospective study on 114 cases. The Veterinary Journal. 205(2): 322-327.

4 Costa C.A., Lunardi Filho W.D. \& Soares N.V. 2003. Assistência humanizada ao cliente oncológico: reflexões junto à equipe. Revista Brasileira de Enfermagem. 56(3): 310-314.

5 Ferreira I., Rahal S.C., Ferreira J. \& Corrêa T.P. 2006. Terapêutica no carcinoma de células escamosas cutâneo em gatos. Ciência Rural. 36(3): 1027-1033.

6 Goldschimidt M.H. \& Hendrick M.J. 2017. Epithelial and Melanocytic Tumors of the Skin In: Meunten D.J. (Ed). Tumor in domestic animals. 5th edn. Ames: John Wiley \& Sons, pp.88-141.

7 Gomes C. \& Witz M.I. 2006. Tratamento com carboplatina intra-tumoral em carcinoma de células escamosas no plano nasal de gatos. Medvep - Revista Cientifica de Medicina Veterinária - Pequenos Animais e Animais de Estimação. 12(4): 132-136.

8 Rigel S.D. \& Gold L.F.S. 2013. The importance of early diagnosis and treatment of actinic keratosis. Journal of the American Academy of Dermatology. 68(1): 17-20.

9 Thomson M. 2007. Squamous cell carcinoma of the nasal planum in cats and dogs. Clinical Techniques in Small Animal Practice. 22(2): 42-45. 